\title{
Radiation effect on free convection flow between oscillating parallel plates with mass diffusion
}

\author{
Fasihah Zulkiflee ${ }^{a}$, Sharidan Shafie ${ }^{a,}$, , Ahmad Qushairi Mohamad a \\ a Department of Mathematics, Faculty of Science, Universiti Teknologi Malaysia, 81310 UTM Johor Bahru, Johor, Malaysia \\ * Corresponding author: sharidan@utm.my
}

\section{Article history}

Received 30 May 2020

Revised 7 September 2020

Accepted 22 September 2020

\begin{abstract}
This study investigates radiation effect on unsteady oscillatory free convection between two parallel plates with mass diffusion. Appropriate non-dimensional variables are used to reduce the dimensional governing equations to dimensionless equation along with imposed initial and boundary conditions. The solutions for velocity, temperature, and concentration profiles are obtained by using Laplace transform method. To illustrate the behavior of the fluid flow, graphical results are presented with influenced of Schmidt number, Prandtl number, radiation parameter, oscillating parameter, Grashof, and mass Grashof number. The corresponding expressions for skin friction, Nusselt number, and Sherwood number are also calculated. It is observed that increasing radiation parameter, Prandtl, and Schmidt number will increase the Nusselt number but the skin friction will be reduced.
\end{abstract}

Keywords: Free convection, radiation, oscillating, parallel plates, mass diffusion

\section{INTRODUCTION}

Convective flows in parallel plates have received much attention due to the wide applications involving natural convection heat transfer [1]. The flow between parallel plates has various application such as in petroleum industry, purification of crude oil, pumps accelerators, and power generators [2]. Many researchers have investigated problem regarding free convection flow between two parallel plates [3-6]. Narahari [7] investigated natural convection flow in vertical channel with ramped wall temperature at one boundary while Jha et al. [8] investigated transient free convective flow in vertical channel with constant temperature and constant heat flux.

Free convection in vertical channel has also been studied with different physical effects such as radiation, heat generation/absorption, chemical reaction, magnetohydrodynamics (MHD), and porous medium [9-12]. Narahari [13] have investigated transient free convection between vertical parallel plates with ramped wall temperature at one boundary with presence of thermal radiation while Jha et al. [14] investigated unsteady natural convection coette flow of heat generating/absorbing fluid between vertical parallel plates filled with porous material. Rajput et al. [15] investigated transient free convection MHD flow between two long vertical parallel plates with constant temperature and variable mass diffusion. The research found that the velocity and skin friction of the fluid increase with increasing the value of time $t$ but decrease with increasing the value of the Prandtl number $P r$, Schmidt number $S c$ and magnetic parameter $M$. Then, Rajput et al. [16] extended his investigation to investigate combined effects of chemical reactions and heat generation/absorption on unsteady transient free convection MHD flow between long vertical parallel plates through a porous medium with constant temperature and mass diffusion.

Oscillatory flow has received some attention in researchers as it was found can higher the rates of heat transfer between parallel plates [17]. Raju et al. [18] investigated unsteady MHD free convection oscillatory Couette flow through a porous medium with periodic wall temperature while Das et al. [19] investigated an oscillatory MHD convective flow in a vertical channel filled with porous medium with hall and thermal radiation effects. While MHD oscillatory flow through a porous channel saturated with porous medium was investigated by Falade et al. [20]. Bunyonyo et al. [17] investigate unsteady oscillatory Coette flow between vertical plates with constant radiative heat flux. The research show that the oscillatory parameter $\omega$ played a greater role causing the fluid to oscillate inside the Coette channel.

Motivated by above investigations, the present analysis is to investigate oscillatory free convection flow between two parallel plates with mass diffusion. Radiation effects is also considered in this research. The problem will be solved using exact method which are Laplace Transform method.

\section{METHODOLOGY}

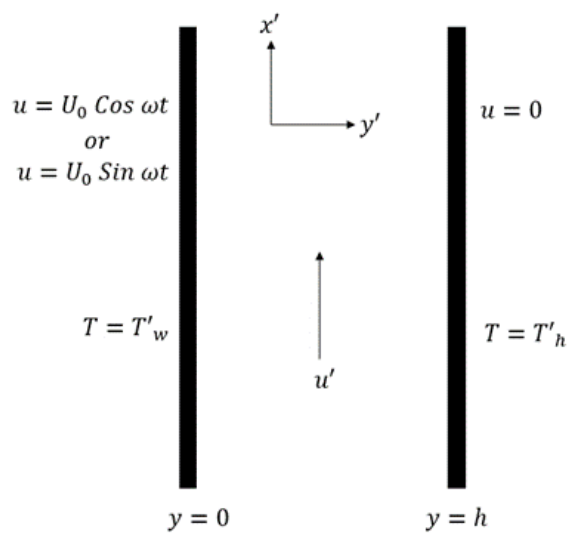

Fig. 1 Flow configuration of the problem.

Let us consider an unsteady free convection flow between two parallel plates with the presence of radiation. Oscillating plate at $y^{\prime}=$ 0 will also be considered in this study. The $x^{\prime}$-axis is considered along one of the vertical plates and the $y^{\prime}$-axis is taken normal to the plates. 
Initially, the time $t^{\prime} \leq 0$, the temperature of the fluid and the plates are same as $T_{h}^{\prime}$ and the concentration of the fluid is $C_{h}^{\prime}$. At $t^{\prime}>0$ the temperature of the plate and concentration of the fluid at $y^{\prime}=0$ are raised to $T_{w}^{\prime}$ and $C_{w}^{\prime}$, respectively, causing the flow of free convection currents. The physical configuration of the problem is presented in Figure 1 and the governing equations under the normal Boussinesq's approximation are as follows:

$\frac{\partial u^{\prime}}{\partial t^{\prime}}=v \frac{\partial^{2} u^{\prime}}{\partial y^{\prime 2}}+g \beta\left(T^{\prime}-T_{h}^{\prime}\right)+g \beta^{*}\left(C^{\prime}-C_{h}^{\prime}\right)$

$\rho C_{\rho} \frac{\partial T^{\prime}}{\partial t^{\prime}}=k \frac{\partial^{2} T^{\prime}}{\partial y^{\prime 2}}-\frac{\partial q_{r}}{\partial y^{\prime}}$

$\frac{\partial C^{\prime}}{\partial t^{\prime}}=D \frac{\partial^{2} C^{\prime}}{\partial y^{\prime 2}}$

$t \leq 0 \quad: u^{\prime}=0 \quad T^{\prime}=T_{h}^{\prime} \quad C^{\prime}=C_{h}^{\prime} \quad$ for $\quad 0 \leq y^{\prime} \leq h$ $t>0: u^{\prime}=U_{0} \cos \omega t$ and $U_{0} \sin \omega t T^{\prime}=T_{W}^{\prime} C^{\prime}=C_{w}^{\prime}$ at $y^{\prime}=0$

$$
u^{\prime}=0 \quad T^{\prime}=T_{h}^{\prime} \quad C^{\prime}=C_{h}^{\prime} \quad \text { at } \quad y^{\prime}=h
$$

where $u^{\prime}$ is velocity of the fluid, $g$ is acceleration due to gravit: the volumetric coefficient of thermal expansion, $t^{\prime}$ is time, $h$ distance between two vertical plates, $T^{\prime}$ is the temperature of the fluid while $T_{h}^{\prime}$ is the temperature of the plate at $y^{\prime}=h, \beta^{*}$ is the volumetric coefficient of concentration expansion, $C^{\prime}$ is the species concentration in the fluid while $C^{\prime} d$ species concentration at $y^{\prime}=h, v$ is the kinematic viscosity, $y^{\prime}$ is the coordinate axis normal to the plates, $\rho$ is the density, $C_{p}^{\prime}$ is the specific heat at constant pressure, $k$ is the thermal conductivity of the fluid, $q_{r}$ is the radiative heat flux, $D$ is mass diffusion coefficient, $T_{w}^{\prime}$ and $C_{w}^{\prime}$ is the temperature and concentration of the plate at $y^{\prime}=0$ and $\omega$ is the frequency of velocity of the wall. by

The radiation heat flux under Rosseland approximation is given

$$
q_{r}=-\frac{4 \sigma^{*}}{3 k^{*}} \frac{\partial T^{4}}{\partial y}
$$

where $\sigma^{*}$ and $k^{*}$ are the Stefan-Boltzamann constant and the mean spectral absorption coefficient, respectively. It is supposed that the temperature difference within the flow are sufficiently small, then equation (5) can be linearized by expanding $T^{4}$ into Taylor series about $T_{h}$, and neglecting higher order terms, we find that

$$
T_{4} \approx 4 T_{h}^{3}-3 T_{h}^{4}
$$

Introducing the following non-dimensional quantities:

$$
\begin{array}{ll}
y=\frac{y^{\prime}}{h} & t=\frac{t^{\prime} v}{h^{2}} \quad u=\frac{u^{\prime}}{U_{0}} \quad T=\frac{T^{\prime}-T_{h}^{\prime}}{T_{w}^{\prime}-T_{h}^{\prime}} \\
C=\frac{C^{\prime}-C_{h}^{\prime}}{C_{w}^{\prime}-C_{h}^{\prime}} & \mu=\rho v
\end{array}
$$

Then, from equation (5)-(7), equations (1)-(3) and boundary conditions (4) becomes:

$$
\begin{gathered}
\frac{\partial u}{\partial t}=\frac{\partial^{2} u}{\partial y^{2}}+G r \theta+G m C \\
\frac{\partial \theta}{\partial t}=\left(\frac{3 R+4}{3 R P r}\right) \frac{\partial^{2} \theta}{\partial y^{2}}
\end{gathered}
$$

$$
\frac{\partial C}{\partial t}=\left(\frac{1}{S c}\right) \frac{\partial^{2} C}{\partial y^{2}}
$$

$$
\begin{aligned}
& t \leq 0: u=0 \quad \theta=0 \quad C=0 \quad \text { for } \quad 0 \leq y \leq 1 \\
& t>0: u=\cos \omega t \text { and } \sin \omega t \quad \theta=1 \quad C=1 \text { at } y=0
\end{aligned}
$$$$
u=0 \quad \theta=0 \quad C=0 \quad \text { at } \quad y=1
$$

where,

$$
\begin{array}{cl}
G r=\frac{g \beta h^{2}}{v U_{0}}\left(T_{w}^{\prime}-T_{h}^{\prime}\right) & G m=\frac{g \beta^{*} h^{2}}{v U_{0}}\left(C_{w}^{\prime}-C_{h}^{\prime}\right) \quad S c=\frac{v}{D} \\
\operatorname{Pr}=\frac{\mu C_{p}}{k} & R=\frac{k k^{*}}{4 \sigma T_{h}^{\prime 3}}
\end{array}
$$

where $G r$ is the thermal Grashof number, $G m$ is the mass Grashof number, $P r$ is the Prandtl number, $S c$ the Schmidt number, $R$ is the radiation parameter, and $\omega$ is the oscillating parameter.

\section{SOLUTIONS OF THE PROBLEM}

The governing equations (8)-(10) with boundary conditions (11) were solved in the exact form by the Laplace transform technique and their solutions in the transform $(y, q)$ plane are given by

$$
\begin{aligned}
\bar{u}_{c}=\sum_{n=0}^{\infty}\left\{\frac{a_{3}}{q^{2}} e^{-a \sqrt{q}}\right. & -\frac{a_{3}}{q^{2}} e^{-b \sqrt{q}}+\frac{1}{2(q-i \omega)} e^{-a \sqrt{q}} \\
& -\frac{1}{2(q-i \omega)} e^{-b \sqrt{q}}+\frac{1}{2(q+i \omega)} e^{-a \sqrt{q}} \\
& -\frac{1}{2(q+i \omega)} e^{-b \sqrt{q}}-\frac{a_{1}}{q^{2}} e^{-a \sqrt{\bar{A} q}}+\frac{a_{1}}{q^{2}} e^{-b \sqrt{\bar{A} q}} \\
& \left.-\frac{a_{2}}{q^{2}} e^{-a \sqrt{s c q}}+\frac{a_{2}}{q^{2}} e^{-b \sqrt{S c q}}\right\} \\
\bar{u}_{s}=\sum_{n=0}^{\infty}\left\{\frac{a_{3}}{q^{2}} e^{-a \sqrt{q}}\right. & -\frac{a_{3}}{q^{2}} e^{-b \sqrt{q}}+\frac{1}{2 i(q-i \omega)} e^{-a \sqrt{q}} \\
& -\frac{1}{2 i(q-i \omega)} e^{-b \sqrt{q}}-\frac{1}{2 i(q+i \omega)} e^{-a \sqrt{q}} \\
& +\frac{1}{2 i(q+i \omega)} e^{-b \sqrt{q}}-\frac{a_{1}}{q^{2}} e^{-a \sqrt{\bar{A} q}}+\frac{a_{1}}{q^{2}} e^{-b \sqrt{\bar{A} q}} \\
& \left.-\frac{a_{2}}{q^{2}} e^{-a \sqrt{S c q}}+\frac{a_{2}}{q^{2}} e^{-b \sqrt{S c q}}\right\} \\
\bar{\theta} & =\sum_{n=0}^{\infty}\left\{\frac{1}{q} e^{-a \sqrt{\bar{A} q}}-\frac{1}{q} e^{-b \sqrt{\bar{A} q}}\right\} \\
\bar{C} & =\sum_{n=0}^{\infty}\left\{\frac{1}{q} e^{-a \sqrt{S c q}}-\frac{1}{q} e^{-b \sqrt{S c q}}\right\} \\
&
\end{aligned}
$$

where the subscripts $c$ and $s$ in above equation refer to cosine and sine oscillations of the plate and

$$
\begin{array}{lcl}
a=2 n+y & b=2 n+2-y & \bar{A}=\frac{3 R P r}{3 R+4} \\
a_{1}=\frac{G r}{\bar{A}-1} & a_{2}=\frac{G m}{S c-1} & a_{3}=a_{1}+a_{2}
\end{array}
$$

Laplace inversion of above equation are as follows: 
$u_{c}=\sum_{n=0}^{\infty}\left\{a_{3}\left(\left(\frac{a^{2}}{2}+t\right) \operatorname{Erfc}\left(\frac{a}{2 \sqrt{t}}\right)-a \sqrt{\frac{t}{\pi}} e^{-\frac{a^{2}}{4 t}}-\left(\frac{b^{2}}{2}+\right.\right.\right.$

$\left.t) \operatorname{Erfc}\left(\frac{b}{2 \sqrt{t}}\right)+b \sqrt{\frac{t}{\pi}} e^{-\frac{b^{2}}{4 t}}\right)+\frac{1}{2}\left(\frac{e^{i \omega t}}{2}\left(e^{a \sqrt{i \omega}} \operatorname{Erfc}\left(\frac{a}{2 \sqrt{t}}+\sqrt{i \omega t}\right)+\right.\right.$ $\left.e^{-a \sqrt{i \omega}} \operatorname{Erfc}\left(\frac{a}{2 \sqrt{t}}-\sqrt{i \omega t}\right)\right)-\frac{e^{i \omega t}}{2}\left(e^{b \sqrt{i \omega}} \operatorname{Erfc}\left(\frac{b}{2 \sqrt{t}}+\sqrt{i \omega t}\right)+\right.$ $\left.\left.e^{-b \sqrt{i \omega}} \operatorname{Erfc}\left(\frac{b}{2 \sqrt{t}}-\sqrt{i \omega t}\right)\right)\right)+\frac{1}{2}\left(\frac{e^{-i \omega t}}{2}\left(e^{a \sqrt{-i \omega}} \operatorname{Erfc}\left(\frac{a}{2 \sqrt{t}}+\right.\right.\right.$ $\left.\sqrt{-i \omega t})+e^{-a \sqrt{-i \omega}} \operatorname{Erfc}\left(\frac{a}{2 \sqrt{t}}-\sqrt{-i \omega t}\right)\right)-$ $\frac{e^{-i \omega t}}{2}\left(e^{b \sqrt{-i \omega}} \operatorname{Erfc}\left(\frac{b}{2 \sqrt{t}}+\sqrt{-i \omega t}\right)+e^{-b \sqrt{-i \omega}} \operatorname{Erfc}\left(\frac{b}{2 \sqrt{t}}-\right.\right.$ $\sqrt{-i \omega t})))+a_{1}\left(-\left(\frac{a^{2} \bar{A}}{2}+t\right) \operatorname{Erfc}\left(\frac{a \sqrt{\bar{A}}}{2 \sqrt{t}}\right)+a \sqrt{\bar{A}} \sqrt{\frac{t}{\pi}} e^{-\frac{a^{2} \bar{A}}{4 t}}+\right.$ $\left.\left(\frac{b^{2} \bar{A}}{2}+t\right) \operatorname{Erfc}\left(\frac{b \sqrt{\bar{A}}}{2 \sqrt{t}}\right)-b \sqrt{\bar{A}} \sqrt{\frac{t}{\pi}} e^{-\frac{b^{2} \bar{A}}{4 t}}\right)+a_{2}\left(\left(\frac{a^{2} S c}{2}+\right.\right.$ t) $\operatorname{Erfc}\left(\frac{a \sqrt{S c}}{2 \sqrt{t}}\right)-a \sqrt{S c} \sqrt{\frac{t}{\pi}} e^{-\frac{a^{2} S c}{4 t}}-\left(\frac{b^{2} S c}{2}+t\right) \operatorname{Erfc}\left(\frac{b \sqrt{S c}}{2 \sqrt{t}}\right)+$ $\left.\left.b \sqrt{S c} \sqrt{\frac{t}{\pi}} e^{-\frac{b^{2} S c}{4 t}}\right)\right\}$

$u_{s}=\sum_{n=0}^{\infty}\left\{a_{3}\left(\left(\frac{a^{2}}{2}+t\right) \operatorname{Erfc}\left(\frac{a}{2 \sqrt{t}}\right)-a \sqrt{\frac{t}{\pi}} e^{-\frac{a^{2}}{4 t}}-\left(\frac{b^{2}}{2}+\right.\right.\right.$ t) $\left.\operatorname{Erfc}\left(\frac{b}{2 \sqrt{t}}\right)+b \sqrt{\frac{t}{\pi}} e^{-\frac{b^{2}}{4 t}}\right)+\frac{1}{2 i}\left(\frac{e^{i \omega t}}{2}\left(e^{a \sqrt{i \omega}} \operatorname{Erfc}\left(\frac{a}{2 \sqrt{t}}+\sqrt{i \omega t}\right)+\right.\right.$ $\left.e^{-a \sqrt{i \omega}} \operatorname{Erfc}\left(\frac{a}{2 \sqrt{t}}-\sqrt{i \omega t}\right)\right)-\frac{e^{i \omega t}}{2}\left(e^{b \sqrt{i \omega}} \operatorname{Erfc}\left(\frac{b}{2 \sqrt{t}}+\sqrt{i \omega t}\right)+\right.$ $\left.\left.e^{-b \sqrt{i \omega}} \operatorname{Erfc}\left(\frac{b}{2 \sqrt{t}}-\sqrt{i \omega t}\right)\right)\right)+\frac{1}{2 i}\left(\frac{e^{-i \omega t}}{2}\left(e^{a \sqrt{-i \omega}} \operatorname{Erfc}\left(\frac{a}{2 \sqrt{t}}+\right.\right.\right.$ $\left.\sqrt{-i \omega t})+e^{-a \sqrt{-i \omega}} \operatorname{Erfc}\left(\frac{a}{2 \sqrt{t}}-\sqrt{-i \omega t}\right)\right)-$ $\frac{e^{-i \omega t}}{2}\left(e^{b \sqrt{-i \omega}} \operatorname{Erfc}\left(\frac{b}{2 \sqrt{t}}+\sqrt{-i \omega t}\right)+e^{-b \sqrt{-i \omega}} \operatorname{Erfc}\left(\frac{b}{2 \sqrt{t}}-\right.\right.$ $\sqrt{-i \omega t})))+a_{1}\left(-\left(\frac{a^{2} \bar{A}}{2}+t\right) \operatorname{Erfc}\left(\frac{a \sqrt{\bar{A}}}{2 \sqrt{t}}\right)+a \sqrt{\bar{A}} \sqrt{\frac{t}{\pi}} e^{-\frac{a^{2} \bar{A}}{4 t}}+\right.$ $\left.\left(\frac{b^{2} \bar{A}}{2}+t\right) \operatorname{Erfc}\left(\frac{b \sqrt{\bar{A}}}{2 \sqrt{t}}\right)-b \sqrt{\bar{A}} \sqrt{\frac{t}{\pi}} e^{-\frac{b^{2} \bar{A}}{4 t}}\right)+a_{2}\left(\left(\frac{a^{2} S c}{2}+\right.\right.$ t) $\operatorname{Erfc}\left(\frac{a \sqrt{S c}}{2 \sqrt{t}}\right)-a \sqrt{S c} \sqrt{\frac{t}{\pi}} e^{-\frac{a^{2} S c}{4 t}}-\left(\frac{b^{2} S c}{2}+t\right) \operatorname{Erfc}\left(\frac{b \sqrt{S c}}{2 \sqrt{t}}\right)+$ $\left.\left.b \sqrt{S c} \sqrt{\frac{t}{\pi}} e^{-\frac{b^{2} S c}{4 t}}\right)\right\}$

$$
\begin{gathered}
\theta=\sum_{n=0}^{\infty}\left\{\operatorname{Erfc}\left(\frac{a \sqrt{\bar{A}}}{2 \sqrt{t}}\right)-\operatorname{Erfc}\left(\frac{b \sqrt{\bar{A}}}{2 \sqrt{t}}\right)\right\} \\
C=\sum_{n=0}^{\infty}\left\{\operatorname{Erfc}\left(\frac{a \sqrt{S c}}{2 \sqrt{t}}\right)-\operatorname{Erfc}\left(\frac{b \sqrt{S c}}{2 \sqrt{t}}\right)\right\}
\end{gathered}
$$

From the solution above, expressions for Skin friction, Nusselt number and Sherwood number were calculated by using the relations,

Skin Friction

Nusselt number

$$
\tau=-\left.\frac{\partial u}{\partial y}\right|_{y=0}
$$

Sherwood number

$$
N u=-\left.\frac{1}{\theta(0, t)} \frac{\partial \theta}{\partial y}\right|_{y=0}
$$

$$
S h=\left.\frac{\partial C}{\partial y}\right|_{y=0}
$$

\section{LIMITING CASES}

In order to underline the theoretical value of the general solutions for velocity, as well to gain physical insight of the flow regime, we consider some special cases whose technical relevance is well-known in the literature.

\section{Solutions for stokes first problem}

By taking $\omega=0$, which corresponds to impulsive motion of the plate, then the velocity yield,

$$
u_{c}=\sum_{n=0}^{\infty}\left\{a _ { 3 } \left(\left(\frac{a^{2}}{2}+t\right) \operatorname{Erfc}\left(\frac{a}{2 \sqrt{t}}\right)-a \sqrt{\frac{t}{\pi}} e^{-\frac{a^{2}}{4 t}}-\left(\frac{b^{2}}{2}+\right.\right.\right.
$$

$\left.t) \operatorname{Erfc}\left(\frac{b}{2 \sqrt{t}}\right)+b \sqrt{\frac{t}{\pi}} e^{-\frac{b^{2}}{4 t}}\right)+\frac{1}{2}\left(\left(\operatorname{Erfc}\left(\frac{a}{2 \sqrt{t}}\right)\right)-\left(\operatorname{Erfc}\left(\frac{b}{2 \sqrt{t}}\right)\right)\right)+$ $\frac{1}{2}\left(\left(\operatorname{Erfc}\left(\frac{a}{2 \sqrt{t}}\right)\right)-\left(\operatorname{Erfc}\left(\frac{b}{2 \sqrt{t}}\right)\right)\right)+a_{1}\left(-\left(\frac{a^{2} \bar{A}}{2}+t\right) \operatorname{Erfc}\left(\frac{a \sqrt{\bar{A}}}{2 \sqrt{t}}\right)+\right.$ $\left.a \sqrt{\bar{A}} \sqrt{\frac{t}{\pi}} e^{-\frac{a^{2} \bar{A}}{4 t}}+\left(\frac{b^{2} \bar{A}}{2}+t\right) \operatorname{Erfc}\left(\frac{b \sqrt{\bar{A}}}{2 \sqrt{\bar{t}}}\right)-b \sqrt{\bar{A}} \sqrt{\frac{t}{\pi}} e^{-\frac{b^{2} \bar{A}}{4 t}}\right)+$ $a_{2}\left(\left(\frac{a^{2} S c}{2}+t\right) \operatorname{Erfc}\left(\frac{a \sqrt{S c}}{2 \sqrt{t}}\right)-a \sqrt{S c} \sqrt{\frac{t}{\pi}} e^{-\frac{a^{2} S c}{4 t}}-\left(\frac{b^{2} S c}{2}+\right.\right.$ $\left.\left.t) \operatorname{Erfc}\left(\frac{b \sqrt{S c}}{2 \sqrt{t}}\right)+b \sqrt{S c} \sqrt{\frac{t}{\pi}} e^{-\frac{b^{2} S c}{4 t}}\right)\right\}$

Respectively,

$$
\begin{aligned}
& u_{s}=\sum_{n=0}^{\infty}\left\{a _ { 3 } \left(\left(\frac{a^{2}}{2}+t\right) \operatorname{Erfc}\left(\frac{a}{2 \sqrt{t}}\right)-a \sqrt{\frac{t}{\pi}} e^{-\frac{a^{2}}{4 t}}-\left(\frac{b^{2}}{2}+\right.\right.\right. \\
& \left.t) \operatorname{Erfc}\left(\frac{b}{2 \sqrt{t}}\right)+b \sqrt{\frac{t}{\pi}} e^{-\frac{b^{2}}{4 t}}\right)+\frac{1}{2 i}\left(\left(\operatorname{Erfc}\left(\frac{a}{2 \sqrt{t}}\right)\right)-\left(\operatorname{Erfc}\left(\frac{b}{2 \sqrt{t}}\right)\right)\right)+ \\
& \frac{1}{2 i}\left(\left(\operatorname{Erfc}\left(\frac{a}{2 \sqrt{t}}\right)\right)-\left(\operatorname{Erfc}\left(\frac{b}{2 \sqrt{t}}\right)\right)\right)+a_{1}\left(-\left(\frac{a^{2} \bar{A}}{2}+t\right) \operatorname{Erfc}\left(\frac{a \sqrt{\bar{A}}}{2 \sqrt{t}}\right)+\right. \\
& \left.a \sqrt{\bar{A}} \sqrt{\frac{t}{\pi}} e^{-\frac{a^{2} \bar{A}}{4 t}}+\left(\frac{b^{2} \bar{A}}{2}+t\right) \operatorname{Erfc}\left(\frac{b \sqrt{\bar{A}}}{2 \sqrt{t}}\right)-b \sqrt{\bar{A}} \sqrt{\frac{t}{\pi}} e^{-\frac{b^{2} \bar{A}}{4 t}}\right)+ \\
& a_{2}\left(\left(\frac{a^{2} S c}{2}+t\right) \operatorname{Erfc}\left(\frac{a \sqrt{S c}}{2 \sqrt{t}}\right)-a \sqrt{S c} \sqrt{\frac{t}{\pi}} e^{-\frac{a^{2} S c}{4 t}}-\left(\frac{b^{2} S c}{2}+\right.\right. \\
& \left.\left.t) \operatorname{Erfc}\left(\frac{b \sqrt{S c}}{2 \sqrt{t}}\right)+b \sqrt{S c} \sqrt{\frac{t}{\pi}} e^{-\frac{b^{2} S c}{4 t}}\right)\right\}
\end{aligned}
$$

2. Solution in the absence of thermal radiation $(R \rightarrow 0)$

In the absence of thermal radiation, the corresponding solutions are directly obtained from the general solutions (8)-(11) by taking $\mathrm{R} \rightarrow 0$.

$$
\begin{aligned}
& u_{c}=\sum_{n=0}^{\infty}\left\{a _ { 3 } \left(\left(\frac{a^{2}}{2}+t\right) \operatorname{Erfc}\left(\frac{a}{2 \sqrt{t}}\right)-a \sqrt{\frac{t}{\pi}} e^{-\frac{a^{2}}{4 t}}-\left(\frac{b^{2}}{2}+\right.\right.\right. \\
& \left.t) \operatorname{Erfc}\left(\frac{b}{2 \sqrt{t}}\right)+b \sqrt{\frac{t}{\pi}} e^{-\frac{b^{2}}{4 t}}\right)+\frac{1}{2}\left(\frac { e ^ { i \omega t } } { 2 } \left(e^{a \sqrt{i \omega}} \operatorname{Erfc}\left(\frac{a}{2 \sqrt{t}}+\sqrt{i \omega t}\right)+\right.\right. \\
& \left.e^{-a \sqrt{i \omega}} \operatorname{Erfc}\left(\frac{a}{2 \sqrt{t}}-\sqrt{i \omega t}\right)\right)-\frac{e^{i \omega t}}{2}\left(e^{b \sqrt{i \omega}} \operatorname{Erfc}\left(\frac{b}{2 \sqrt{t}}+\sqrt{i \omega t}\right)+\right. \\
& \left.\left.e^{-b \sqrt{i \omega}} \operatorname{Erfc}\left(\frac{b}{2 \sqrt{t}}-\sqrt{i \omega t}\right)\right)\right)+\frac{1}{2}\left(\frac { e ^ { - i \omega t } } { 2 } \left(e ^ { a \sqrt { - i \omega } } \operatorname { E r f c } \left(\frac{a}{2 \sqrt{t}}+\right.\right.\right. \\
& \left.\sqrt{-i \omega t})+e^{-a \sqrt{-i \omega}} \operatorname{Erfc}\left(\frac{a}{2 \sqrt{t}}-\sqrt{-i \omega t}\right)\right)- \\
& \frac{e^{-i \omega t}}{2}\left(e^{b \sqrt{-i \omega}} \operatorname{Erfc}\left(\frac{b}{2 \sqrt{t}}+\sqrt{-i \omega t}\right)+e^{-b \sqrt{-i \omega}} \operatorname{Erfc}\left(\frac{b}{2 \sqrt{t}}-\right.\right. \\
& \sqrt{-i \omega t})))+a_{1}\left(-\left(\frac{a^{2} P r}{2}+t\right) \operatorname{Erfc}\left(\frac{a \sqrt{P r}}{2 \sqrt{t}}\right)+a \sqrt{\operatorname{Pr}} \sqrt{\frac{t}{\pi}} e^{-\frac{a^{2} P r}{4 t}}+\right. \\
& \left.\left(\frac{b^{2} P r}{2}+t\right) \operatorname{Erfc}\left(\frac{b \sqrt{P r}}{2 \sqrt{t}}\right)-b \sqrt{\operatorname{Pr}} \sqrt{\frac{t}{\pi}} e^{-\frac{b^{2} P r}{4 t}}\right)+a_{2}\left(\left(\frac{a^{2} S c}{2}+\right.\right.
\end{aligned}
$$


t) $\operatorname{Erfc}\left(\frac{a \sqrt{S c}}{2 \sqrt{t}}\right)-a \sqrt{S c} \sqrt{\frac{t}{\pi}} e^{-\frac{a^{2} S c}{4 t}}-\left(\frac{b^{2} S c}{2}+t\right) \operatorname{Erfc}\left(\frac{b \sqrt{S c}}{2 \sqrt{t}}\right)+$ $\left.\left.b \sqrt{S c} \sqrt{\frac{t}{\pi}} e^{-\frac{b^{2} S c}{4 t}}\right)\right\}$

$u_{s}=\sum_{n=0}^{\infty}\left\{a_{3}\left(\left(\frac{a^{2}}{2}+t\right) \operatorname{Erfc}\left(\frac{a}{2 \sqrt{t}}\right)-a \sqrt{\frac{t}{\pi}} e^{-\frac{a^{2}}{4 t}}-\left(\frac{b^{2}}{2}+\right.\right.\right.$

$\left.t) \operatorname{Erfc}\left(\frac{b}{2 \sqrt{t}}\right)+b \sqrt{\frac{t}{\pi}} e^{-\frac{b^{2}}{4 t}}\right)+\frac{1}{2 i}\left(\frac{e^{i \omega t}}{2}\left(e^{a \sqrt{i \omega}} \operatorname{Erfc}\left(\frac{a}{2 \sqrt{t}}+\sqrt{i \omega t}\right)+\right.\right.$

$\left.e^{-a \sqrt{i \omega}} \operatorname{Erfc}\left(\frac{a}{2 \sqrt{t}}-\sqrt{i \omega t}\right)\right)-\frac{e^{i \omega t}}{2}\left(e^{b \sqrt{i \omega}} \operatorname{Erfc}\left(\frac{b}{2 \sqrt{t}}+\sqrt{i \omega t}\right)+\right.$

$\left.\left.e^{-b \sqrt{i \omega}} \operatorname{Erfc}\left(\frac{b}{2 \sqrt{t}}-\sqrt{i \omega t}\right)\right)\right)+\frac{1}{2 i}\left(\frac{e^{-i \omega t}}{2}\left(e^{a \sqrt{-i \omega}} \operatorname{Erfc}\left(\frac{a}{2 \sqrt{t}}+\right.\right.\right.$

$\left.\sqrt{-i \omega t})+e^{-a \sqrt{-i \omega}} \operatorname{Erfc}\left(\frac{a}{2 \sqrt{t}}-\sqrt{-i \omega t}\right)\right)-$

$\frac{e^{-i \omega t}}{2}\left(e^{b \sqrt{-i \omega}} \operatorname{Erfc}\left(\frac{b}{2 \sqrt{t}}+\sqrt{-i \omega t}\right)+e^{-b \sqrt{-i \omega}} \operatorname{Erfc}\left(\frac{b}{2 \sqrt{t}}-\right.\right.$

$\sqrt{-i \omega t})))+a_{1}\left(-\left(\frac{a^{2} P r}{2}+t\right) \operatorname{Erfc}\left(\frac{a \sqrt{P r}}{2 \sqrt{t}}\right)+a \sqrt{\operatorname{Pr}} \sqrt{\frac{t}{\pi}} e^{-\frac{a^{2} P r}{4 t}}+\right.$

$\left.\left(\frac{b^{2} P r}{2}+t\right) \operatorname{Erfc}\left(\frac{b \sqrt{P r}}{2 \sqrt{t}}\right)-b \sqrt{P r} \sqrt{\frac{t}{\pi}} e^{-\frac{b^{2} P r}{4 t}}\right)+a_{2}\left(\left(\frac{a^{2} S c}{2}+\right.\right.$

t) $\operatorname{Erfc}\left(\frac{a \sqrt{S c}}{2 \sqrt{t}}\right)-a \sqrt{S c} \sqrt{\frac{t}{\pi}} e^{-\frac{a^{2} S c}{4 t}}-\left(\frac{b^{2} S c}{2}+t\right) \operatorname{Erfc}\left(\frac{b \sqrt{S c}}{2 \sqrt{t}}\right)+$ $\left.\left.b \sqrt{S c} \sqrt{\frac{t}{\pi}} e^{-\frac{b^{2} S c}{4 t}}\right)\right\}$

\section{RESULTS AND DISCUSSION}

In this section, the obtained exact solutions are studied numerically in order to determine the effects of several involved parameter such as Prandtl number Pr, Grashof number Gr, Mass Grashof number Gm, Schmidt number Sc, radiation parameter R, and oscillating parameter $\omega$. Skin friction, Nusselt number, and Sherwood number are also plotted with different parameters.

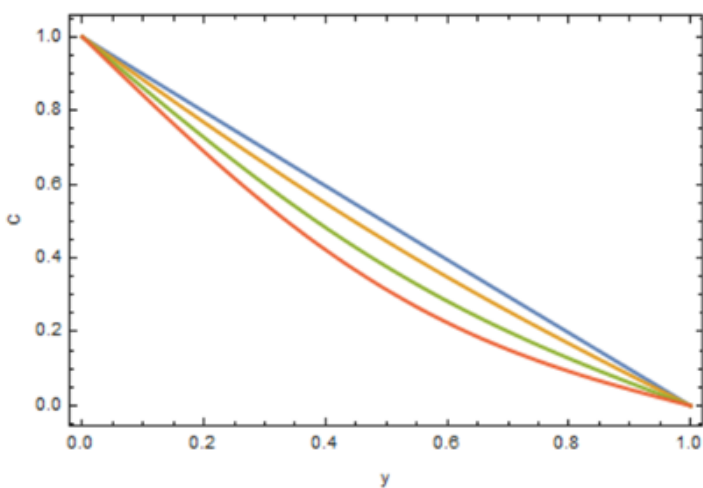

Fig. 2 Concentration profile with different $S c$ number with $t=0.1$.

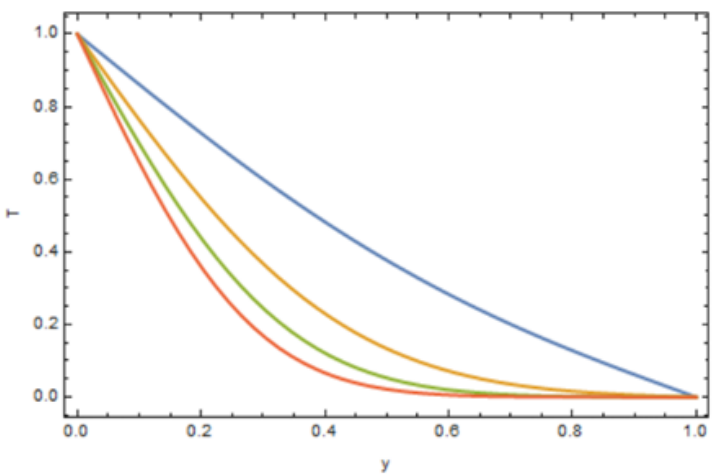

Fig. 3 Temperature profile with different $\operatorname{Pr}$ number with $t=0.1$

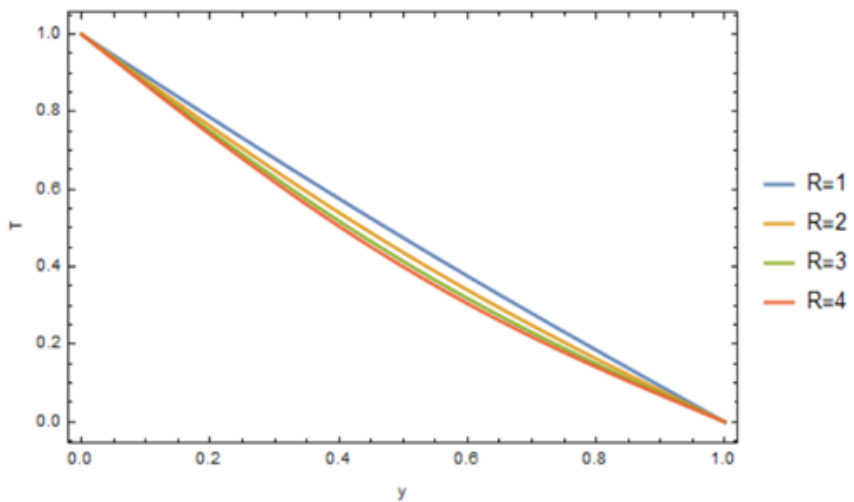

Fig. 4 Temperature profile with different $R$ number with $t=0.1$ and $P r=$ 0.71

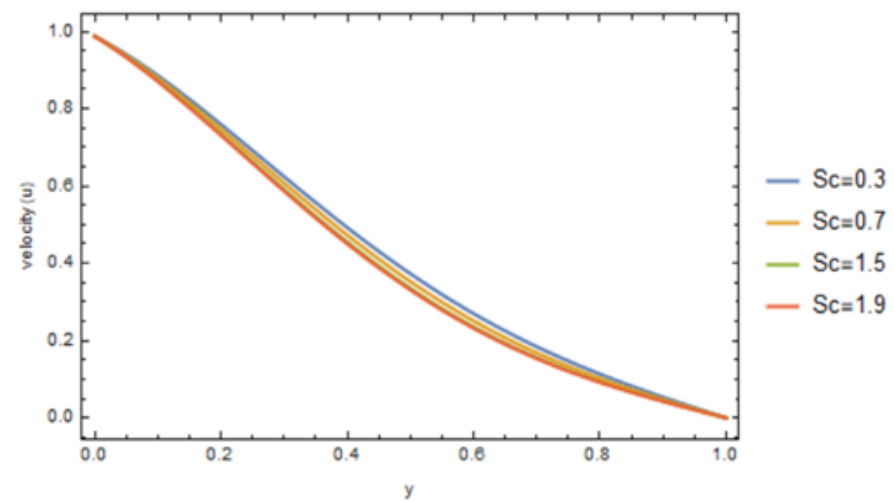

Fig. 5 Velocity profile with different $S c$ number with $t=0.1, \operatorname{Pr}=3, G r=$ $2, \mathrm{Gm}=2, \omega=\frac{\pi}{2}, R=0.2$

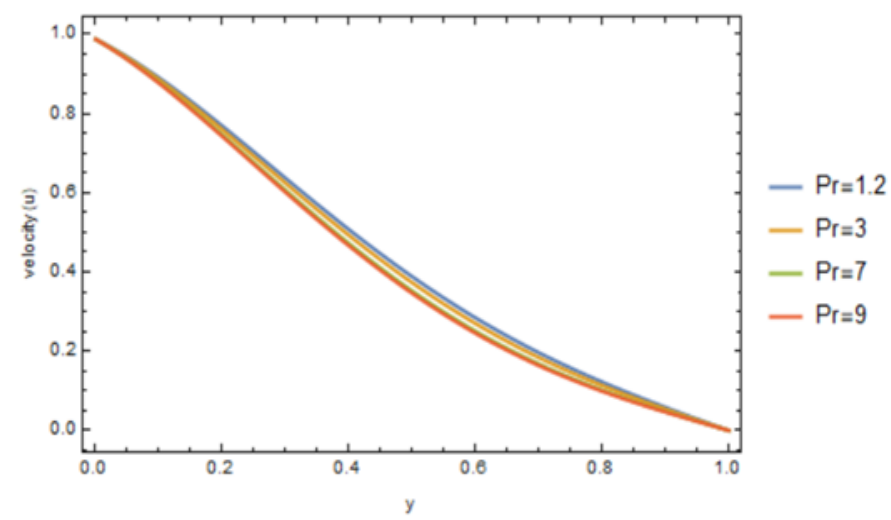

Fig. 6 Velocity profile with different $\operatorname{Pr}$ number with $t=0.1, S c=0.3$, $G r=2, G m=2, \omega=\frac{\pi}{2}, R=0.2$

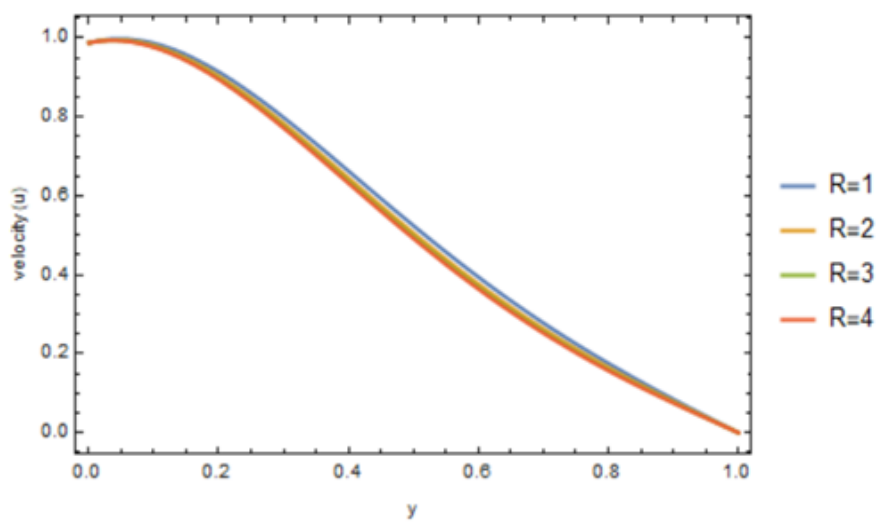

Fig. 7 Velocity profile with different $R$ number with $t=0.1, \operatorname{Pr}=0.71$, $G r=5, G m=5, \omega=\frac{\pi}{2}, S c=0.5$ 


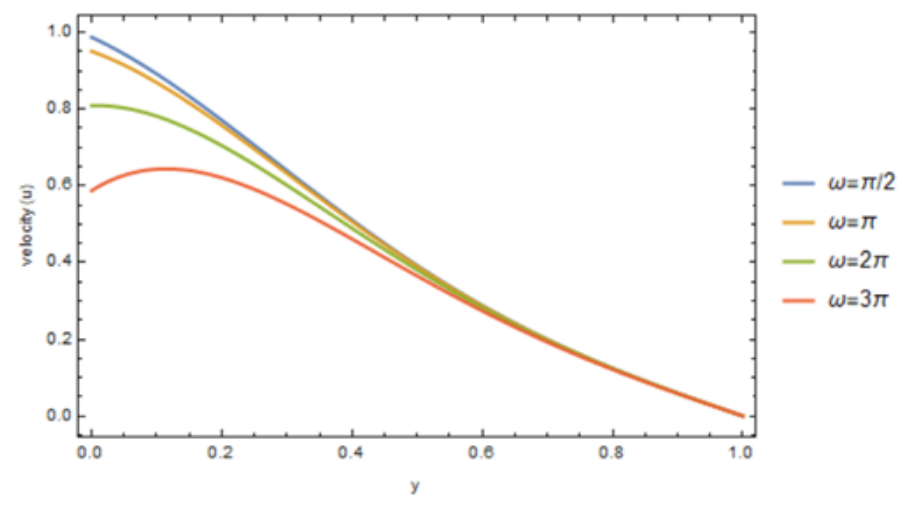

Fig. 8 Velocity profile with different $\omega$ number with $t=0.1, S c=0.3, G r=$ 2, $G m=2, \operatorname{Pr}=0.71, R=0.2$

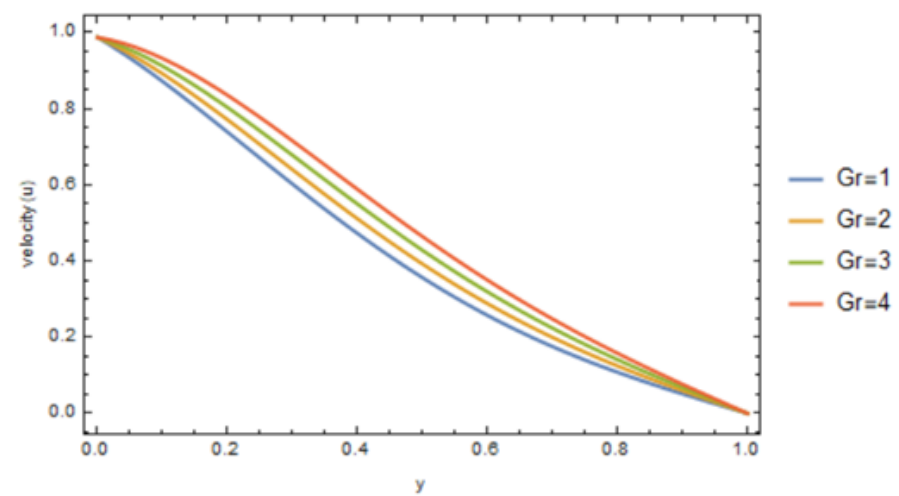

Fig. 9 Velocity profile with different $G r$ number with $t=0.1, \operatorname{Pr}=0.71$, $G r=2, G m=2, \omega=\frac{\pi}{2}, R=0.2$

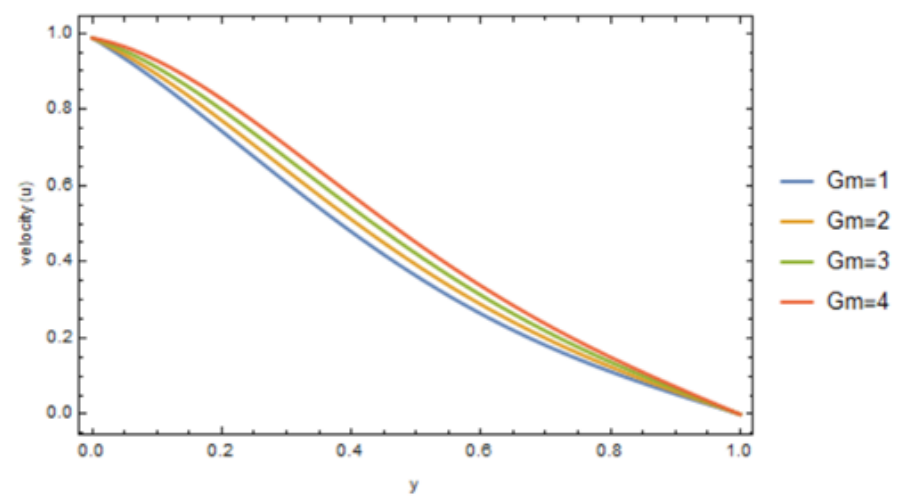

Fig. 10 Velocity profile with different $G m$ number with $t=0.1, S c=0.3$, Gr $=2, \omega=\frac{\pi}{2}, \operatorname{Pr}=0.71, R=0.2$

Table 1 Skin friction with different parameter.

\begin{tabular}{l|llllcll}
$\mathbf{t}$ & $\mathbf{P r}$ & $\mathbf{S c}$ & $\mathbf{G r}$ & $\mathbf{G m}$ & $\boldsymbol{\omega}$ & $\mathbf{R}$ & \multicolumn{1}{c}{$\boldsymbol{\tau}$} \\
\hline $\mathbf{0 . 1}$ & 0.71 & 0.3 & 2 & 2 & $\pi$ & 2 & -0.663458 \\
$\mathbf{0 . 1}$ & 0.71 & 0.3 & 2 & 2 & $2 \pi$ & 2 & 0.00182304 \\
$\mathbf{0 . 1}$ & 0.71 & 0.3 & 2 & 2 & $3 \pi$ & 2 & 1.01048 \\
$\mathbf{0 . 1}$ & 3 & 0.3 & 2 & 2 & $2 \pi$ & 2 & -0.124196 \\
$\mathbf{0 . 1}$ & 5 & 0.3 & 2 & 2 & $2 \pi$ & 2 & -0.167746 \\
$\mathbf{0 . 1}$ & 3 & 0.3 & 2 & 2 & $2 \pi$ & 1 & -0.0945283 \\
$\mathbf{0 . 1}$ & 3 & 0.3 & 2 & 2 & $2 \pi$ & 3 & -0.136619
\end{tabular}

Table 2 Nusselt number with different parameter.

\begin{tabular}{l|lll}
$\mathbf{t}$ & $\mathbf{P r}$ & $\mathbf{R}$ & $\mathbf{N u}$ \\
\hline $\mathbf{0 . 1}$ & 0.71 & 2 & 1.18092 \\
$\mathbf{0 . 1}$ & 3 & 2 & 2.39365 \\
$\mathbf{0 . 1}$ & 5 & 2 & 3.0919 \\
$\mathbf{0 . 1}$ & 0.71 & 1 & 1.0311 \\
$\mathbf{0 . 1}$ & 0.71 & 3 & 1.26002
\end{tabular}

Table 3 Sherwood number with different Sc parameter.

\begin{tabular}{c|cc}
$\mathbf{t}$ & Sc & Sh \\
\hline $\mathbf{0 . 1}$ & 0.3 & 1.02586 \\
$\mathbf{0 . 1}$ & 0.5 & 1.27007 \\
$\mathbf{0 . 1}$ & 0.7 & 1.49407
\end{tabular}

Figure 2 shows concentration profiles graph with different Schmidt $(S c)$ number. From the graph, increasing the $S c$ number will reduce the concentration. While Figures 3 and 4 show temperature profile against Prandtl $(\mathrm{Pr})$ number and radiation parameter. It shows that increasing $\mathrm{Pr}$ and Radiation parameter will reduce the temperature of the fluid flow. Physically, due to high number of $\mathrm{Pr}$ number will cause the thermal conductivity of the fluid decreases and causing the viscosity of the fluid increases that would make the temperature to decrease. Besides, when radiation is present, the thermal boundary layer was always found to thicken which may be explained that radiation provides additional means to diffused energy that causing the temperature to decreases.

Figure 5-10 show the effects of different parameters on the velocity profile. From Figure 5, it is observed that increasing $S c$ number will decrease the velocity. It is found that $S c$ number relates to the relative thickness of the mass transfer boundary layer, if $S c$ number is increasing, the thickness of boundary layer would increase and will cause the velocity decreases. While Figure 6 and 7 show the velocity profile with different $\mathrm{Pr}$ and radiation parameter. Increasing both $\mathrm{Pr}$ and radiation parameter will cause the velocity to decreases. Figure 8 show velocity profile for different oscillation parameter. The graph shows that increasing the oscillation parameter will cause the velocity to decrease. While Figure 9 and 10 show velocity field with different Grashof number $(\mathrm{Gr})$ and mass Grashof number $(\mathrm{Gm})$. It is observed that velocity increasing with increasing the value of $G r$. Physically this is possible because as $\mathrm{Gr}$ number increases, the contribution from the buoyancy force near the plate become significant and hence a rise in velocity is observed. Similar to mass Grashof number, when $\mathrm{Gm}$ is increased, the velocity was found increased.

Table 1 shows the skin friction for different $P r$ and radiation parameter. It is depicted from the table that increasing $P r$ and Radiation parameter causes the skin friction to decrease. Nusselt number with different $\operatorname{Pr}$ and radiation parameter are presented in Table 2. It shows that increasing the $P r$ and radiation will increase the Nusselt number. While Table 3 shows solution of Sherwood number against time with different $S c$ number. The table shows that increasing the $S c$ number will increase the Sherwood number.

\section{CONCLUSION}

An exact solution to the problem of oscillating free convection flow between two parallel plates with mass diffusion and radiation effects is investigated. Solutions of the problem are obtained by using Laplace transform technique. The effects of various parameters on velocity, temperature and concentration profiles as well as skin friction, Nusselt and Sherwood number are numerically studied. The following conclusions can be summarized from this study.

- Increasing Schmidt number will reduce the concentration of the fluid but increase the Sherwood number.

- Increasing Prandtl number will reduce the temperature, velocity and skin friction but will increase the Nusselt number.

- Increasing the oscillation parameter will lower the velocity.

- Increasing radiation parameter will reduce the temperature, velocity, and skin friction but will increase the Nusselt number.

- Increasing Grashof and mass Grashof number will increase the velocity of the fluid flow.

- The solutions satisfy the initial and boundary conditions.

\section{ACKNOWLEDGEMENT}

The authors would like to acknowledge Ministry of Higher Education (MOE) UTM Zamalah and Research Management Centre- 
UTM, Universiti Teknologi Malaysia (UTM) for the financial support through vote numbers 5F004, 07G70, 07G72, 07G76 and 07G77 for this research.

\section{REFERENCES}

[1] Al-subaie, M. A., \& Chamkha, A. J. (2004). Transient natural convection flow of a particulate suspension through a vertical channel 40 707-713.

[2] Makinde, O. D., \& Mhone, P. Y. (2005). Heat transfer to MHD oscillatory flow in a channel filled with porous medium. Romanian Journal of physics, 50(9/10), 931

[3] Kundu, B. (2015). Semianalytical methods for heat and fluid flow between two parallel plates. Journal of Thermal Engineering, 1(3), 175-181.

[4] Hamza, M. M. (2016). Free convection slip flow of an exothermic fluid in a convectively heated vertical channel. Ain Shams Engineering Journal.

[5] Singh, A. K., Gholami, H. R., \& Soundalgekar, V. M. (1996). Transient free convection flow between two vertical parallel plates. Heat and mass transfer, 31(5), 329-331.

[6] Singh, A. K., \& Paul, T. (2006). Transient natural convection between two vertical walls heated/cooled asymmetrically. International Journal of Applied Mechanics and Engineering, 11(1), 143-154.

[7] Narahari, M. (2010). Effects of thermal radiation and free convection currents on the unsteady Couette Flow between two vertical parallel plates with constant heat flux at one boundary. Wseas Transactions on heat and mass transfer 5(1) 21-30.

[8] Paul, T., Jha, B. K., \& Singh, A. K. (1996). Transient free convective flow in a vertical channel with constant temperature and constant heat flux on walls. Heat and mass transfer, 32(1-2), 61-63. 1

[9] Singh, A. K. (2018). Effect of Newtonian Cooling/Heating on MHD Free Convective Flow between Vertical Walls with Induced Magnetic Field. In Applications of Fluid Dynamics (pp. 411-430). Springer, Singapore.

[10] K. Jha, B., \& Isa, S. (2013). Computational Treatment of MHD Transient Natural Convection Flow in a Vertical Channel Due to Symmetric Heating in Presence of Induced Magnetic Field. Journal of the physical society of Japan, 82(8), 084401.

[11] Seth, G. S., Singh, J. K., \& Mahato, G. K. (2012). Effects of Hall current and rotation on unsteady hydromagnetic Couette flow within a porous channel. International Journal of Applied Mechanics, 4(02), 1250015.
[12] Alagoa, K. D., Tay, G., \& Abbey, T. M. (1998). Radiative and free convective effects of a MHD flow through a porous medium between infinite parallel plates with time-dependent suction. Astrophysics and Space Science, 260(4), 455-468.

[13] Narahari, M. Transient free convection Flow between long vertical parallel plates with ramped Wall temperature at one boundary in the presence of thermal radiation and constant mass diffusion. Meccanica 47(8) (2012), 1961-1976.

[14] Jha, B. K., \& Musa, M. K. (2012). Unsteady natural convection Couette flow of heat generating/absorbing fluid between vertical parallel plates filled with porous material. Applied Mathematics and Mechanics, 33(3), 303-314.

[15] Rajput, U. S., \& Sahu, P. K. (2011). Transient free convection MHD flow between two long vertical parallel plates with constant temperature and variable mass diffusion. Journal of Math. Analysis, 34(5), 1665-1671.

[16] Rajput, U. S., \& Sahu, P. K. (2011). Combined effects of chemical reactions and heat generation/absorption on unsteady transient free convection MHD flow between two long vertical parallel plates through a porous medium with constant temperature and mass diffusion. Elixir Appl. Math, 39, 4855-4859.

[17] Bunonyo, K. W., Amos, E., \& Eli, I. C. (2018). Unsteady Oscillatory Couette Flow between Vertical Parallel Plates with Constant Radiative Heat Flux.

[18] Varma, S., \& Raju, M. (2011). Unsteady MHD free convection oscillatory Couette flow through a porous medium with periodic wall temperature.

[19] Das, S., Jana, R. N., \& Makinde, O. D. (2014). An oscillatory MHD convective flow in a vertical channel filled with porous medium with Hall and thermal radiation effects. Special Topics \& Reviews in Porous Media: An International Journal, 5(1).

[20] Falade, J. A., Ukaegbu, J. C., Egere, A. C., \& Adesanya, S. O. (2017). MHD oscillatory flow through a porous channel saturated with porous medium. Alexandria engineering journal, 56(1), 147-152. 